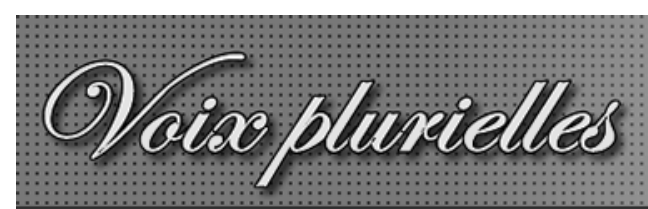

Revue de l'Association des Professeur-e-s de Français des Universités et Collèges Canadiens

\author{
Voix plurielles \\ Volume 3, Numéro 2 : septembre 2006
}

Georges Farid

\title{
La violence verbale entre catholiques et protestants au XVIe siècle
}

Citation MLA : Farid, Georges. «La violence verbale entre catholiques et protestants au XVIe siècle .» Voix plurielles 3.2 (septembre 2006).

(c) Voix plurielles, revue électronique de l'APFUCC 2006. 


\title{
La violence verbale entre catholiques et protestants au XVIe siècle
}

\author{
Georges Farid \\ Université
}

Septembre 2006

$\mathrm{N}$ otre étude descriptive porte sur les diverses formes sous lesquelles se présentent la violence verbale. Nous nous sommes arrêté particulièrement sur les procédés littéraires d'attaque entre catholiques et protestants au XVIe siècle. Notre objectif est de présenter les expressions injurieuses utilisées afin d'en saisir la portée et leur raison d'être ou « d'avoir été ».

Il ne s'agit point d'identifier qui est le champion de l'expression injurieuse mais bien de décrire simplement les termes en question et d'interpréter ce que traduisent ces paroles véhémentes : incompréhension de l'autre, rejet, haine. Y aurait-il une leçon à tirer dans l'utilisation d'un tel langage qui nous offre toute la latitude d'humilier les autres par l'expression la plus vulgaire (mots grossiers) ou l'expression la plus subtile (ironie, sarcasme).

\section{Bref aperçu des hostilités de la période 1510-1584 et fonction sociale des insultes}

Postel (2004) note que « les historiens d'aujourd'hui insistent beaucoup sur le fait que l'ébranlement religieux du XVIe siècle refléterait une crise spirituelle plus profonde [...] que celle résultant d'une simple dénonciation des tares de l'Église romaine. Siècle d'angoisse eschatologique du peuple catholique, dit-on, à quoi la Réforme aurait opposé son entreprise de désangoissement » (17). Ce point de vue est celui que soutient Crouzet (1990) qui interprète qu'entre 1560-1570 c'est le temps de déchaînement de deux violences : l'une, catholique, déterminée par une pulsion mystique et l'autre, huguenote, par une rationalité de l'action (mettre en oeuvre sa conversion).

Lécrivain (1997) fait remarquer que « préparée lointainement par un désir général de réforme qu'exprimèrent bientôt, selon des contours incertains, les réformations catholique et protestante, cette période est celle des guerres de religion [...] Nulle part ailleurs en Europe, les troubles n'ont été aussi intenses et n'ont duré si longtemps ». Lécrivain résume cette période (traditionnellement entre 1560 et 1610) comme «l'angoisse devant un monde qui s'affronte et le refus d'une monarchie corrompue, contre la volonté d'instaurer une société sainte et sans hiérarchie ».

Malgré l'existence de respectables prélats, de curés et de pieux moines chez les catholiques, il reste que les réformés sont à la recherche d'un christianisme plus proche de Dieu, christianisme qu'ils veulent débarrassé des scories d'un clergé dont l'image est, en grande partie, détériorée par ses perversions déjà condamnées par les conciles et par les censeurs catholiques mêmes; ces perversions et les tares visibles de l'Église seront alors plus efficacement dénoncées par Luther et 
Calvin.

Les livres « réformés » viennent en particulier de Suisse. Dès lors, entre 1510 et 1534, on passe des premiers contestataires à la violente dénonciation et, par la suite, de la dissension à la crise. De 1535 à 1549, c'est la construction doctrinale protestante et la répression royale. Les luthériens autrefois poursuivis sont amnistiés ; ceux qui propageront « l'hérésie » seront pendus.

Au début du siècle, c'est l'ironie que les réformés décochent aux catholiques, ironie qui se transforme en insulte utilisée, selon Postel, comme armes « visant hommes de foi, pratiques et institutions religieuses, entités politiques [...]». Les textes polémiques ont alors comme fonction de « provoquer un mouvement d'opinion en dévalorisant, en déshumanisant l'adversaire par l'ironie, le mépris, l'insulte » (18). En effet, ce qui est perçu comme simple moquerie, au départ par les catholiques, deviendra menace pour ceux-ci étant donné les fêlures déjà nombreuses dans leurs moeurs religieuses; d'où les répliques verbales et textuelles ultérieures qui s'accompagneront de persécutions contre les réformés.

Sans prétendre avoir parfaitement saisi la fonction sociale de l'insulte au XVIe siècle, par le bref aperçu du contexte précédent, nous pouvons mieux mesurer l'impact et la portée des insultes entre catholiques et protestants où le « verbe » attisera et fera couler le « sang ».

\section{Injures, insultes ou invectives?}

Avant d'aborder notre étude, il importe de clarifier certaines notions dont il sera question, à savoir la définition des termes « injures, insultes, invectives ». Le Petit Robert (1993), sous le vocable « injure », donne quatre définitions que nous résumons comme suit :

1- Injustice, traitement injuste. Faire tort (sens vieilli).

2- $\quad$ Dommage causé par les éléments, le temps. L'injure des ans, du sort (sens littéraire).

3- Offense grave et délibérée (sens littéraire et vieilli). Voir « affront, avanie, insulte, outrage ». [...] Faire injure : offenser.

4- Parole offensante (sens courant). Voir « attaque, calomnie, insolence, insulte, invective, sottise ». [...] injures grossières. [...] Délit consistant à proférer à l'encontre de quelqu'un un terme de mépris. Voir « outrage ». Bénac (1956) ajoute « offense de fait et surtout de parole ou par écrit toujours faite à dessein et blessant gravement $»$.

En ce qui concerne « insulte » : acte ou parole qui vise à outrager ou qui constitue un outrage ; le Petit Robert nous réfère à « affront, injure, offense ». Bénac clarifie " injure dans le dessein prémédité de traiter insolemment, avec dérision ou mépris, souvent du grand ou petit ».

Pour « invective » : parole ou suite de paroles violentes contre quelqu'un ou quelque chose; le Petit Robert nous renvoie ensuite à « injure, insulte ». Bénac note que ce terme est généralement employé au pluriel pour signifier « reproches dirigés contre les personnes ou les choses morales et surtout outrageants par la façon passionnée dont ils sont proférés ».

Par rapport à « offense » : parole ou action qui offense, qui blesse quelqu'un dans son honneur, 
dans sa dignité; Bénac précise « fait ou parole qui blesse la dignité de quelqu’un, volontairement ou non ».

Les termes qui reflèteraient le mieux les expressions injurieuses dans la polémique entre catholiques et protestants sont « invectives, injures » au sens courant.

\section{Invectives dans la polémique religieuse en France au XVIe siècle entre catholiques et protestants}

Dans la crise politico-religieuse qui a ensanglanté la France entre 1510 et 1580, nous citerons des passages de Postel (2004) assez révélateurs où s'enchevêtrent injures, mépris et ironie dont le but est de provoquer un mouvement d'opinion non sans dévaloriser et déshumaniser l'adversaire. Dans la plupart des cas, nous avons conservé l'orthographe originale des passages cités ; il arrive qu'un auteur orthographie un même mot différemment dans une même ligne.

Une des manières que les réformés (les protestants) adoptaient consistait à écrire des pamphlets où l'irrespect à l'égard du pape et de son clergé était bien évident. Une autre consistait à écrire des livres comme Le Baston pour chasser les loups censé être l'un des premiers livres protestants dans lequel on peut lire que dans le clergé : « Les uns sont apostats infâmes, sacrilèges et hérétiques. Les autres putiers et bigames, mariez, tous apostatiques (= apostoliques) » (45).

La riposte des catholiques, dans un style combattant, se lit dans un petit livret de 60 feuillets Contre les ténébrions, lumière évangélique sous la plume de Jérôme de Hangest, vicaire général du Mans : «Ces abominables ténébrions... insidiatifs pirates... pervers calomniateurs » que sont les disciples de Luther (57).

Les polémistes des deux camps décochent leurs flèches verbales où la vulgarité s'allie à l'ironie. Calvin, dans L'institution de la religion chrétienne expose « la venimeuse iniquité des calomniateurs » et pose la question «Qu'est-ce que le monde honore aujourd'huy en ces Evesques cornuz... De ces hérétiques rebelles et contumax sont issus tous les Papes, Cardinaux, Evesques, Abbez et Prestres ». Quant aux cloîtres, ce sont des « bordeaux » où se rassemblent les « ventres oysifz des moynes » (67).

Luther, dans son Antithèse de la vray et fausse Église, s'en prend à ce qu'il appelle « les petites paillardes nouvellement appaillardées » : les nonnes (76).

Face à Calvin et à Luther s'élève un moine, Artus Désiré qui va mener la contre-offensive catholique avec son Miroir des Francs-Taulpins publié en 1546 ; c'est ainsi qu'il caractérise Luther comme un « esprit damné... homme orgueilleux, plus fier que l'éléphant, producteur d'infernale vermine ... » et l'interpelle en lui disant « Respons, putain qui fais aux sainctz la guerre... »; quant à ses disciples, Désiré les appelle

« ... Luthériens, malheureux réprouvez ... idiotz ... (80). Ce discours catholique est ponctué d'appels à l'extermination des hérétiques : « Ne doit-on pas faire cendre... sans les punir par amende honorable ?... faire feu de vos oz ... Et vous brûler comme gens idiotz... » (80).

Calvin, dans son ouvrage de 1550 Des scandales qui empeschent aujourdhuy beaucoup de gens de venir à la pure doctrine de l'Évangile et en desbauchent d'autres, s'en prend, entre autres, aux moines : " ventres paresseux, mutins, traistres et desloyaux, ingratz, adonnez à picques et à faire bandes, larrons, de nature servile et paillards... (88). Calvin, en critiquant le célibat des prêtres, poursuit: «Ces jolis puceaux gardent leurs licts vuydes d'une seule femme afin qu'ils ayent congé 
d'envahir tous les litz des gens mariez... » (88).

Théodore de Bèze, futur successeur de Calvin, dans son Epistre de Maistre Benoit Passavant à Messire Pierre Lizet (président du Parlement de Paris qui a la charge des poursuites contre l'hérésie) traite Lizet de « cochon... mulet... misérable bestiole... » et s'en prend avec vulgarité à l'Église : « Notre Sainte Mère putain l'Église romaine... catin, archi-catin qui écarte les jambes sous tous les arbres » et dont les « Papes sodomites » (118), à « l'humanité cacaturiante » (36) sont « du genre épicène » (42)...; « les Évangiles de l'Antéchrist... sont des excréments que le Diable a vomi par [leur] bouche fétide [car] bouche du Pape et cul du Diable c'est tout un » (48). Mais que ceux qui ne veulent écouter que la Vraie Parole ne se découragent pas, car « ...voici venir le temps où putain périra avec ses maquereaux » (101).

Comment réagit la polémique catholique, trois ans après les invectives de Théodore de Bèze ? Antoine Cathelan, dans son Passevent, parisien, répondant à un Pasquin, Rommain, attaque individuellement les Genevois en question : «Hommes détestables que Calvin... et l'abominable Viret qui, après qu'ils sont soulz..., disent de ceux qui se font brusler qu'ilz sont comme moutons (3) ; qui dans leurs sermons, appellent « le Pape Antéchrist et les Cardinaux cuisiniers et les Prestres et Moynes vermines et cafards » (19); qui, par la bouche de Viret, " ce grand paillard de l'Église » (23), appellent la messe : la vesse. « Je ne doute point, écrit Cathelan, que leurs Églises soient pires que des étables de pourceaux... Vailler (compagnon de Viret)... a étudié auprès d'une vieille midrouille (25), sa paillarde ; Jean Rubite, le grand théologien de Lausanne, qui, en chaire, pour chasser les poulx et morpions a la braguette délacée ou la main dedans (28). Tout ce monde vit une vie dissolue. Bèze engrosse une chambrière qu'il fait avorter ; on lui connaît des amours avec la « la belle Candide », tenancière de bordel « bien puante et musquée » (30). Calvin, « le grand Satrapus $\gg \ldots »(105)$.

Avec l'admission des jésuites en France, leur général Diego Lainez, successeur d'Ignace de Loyola, qualifie les protestants, selon la terminologie en vigueur, de « loups, renards, serpents et assassins » (141).

En 1567, paraît un ouvrage contre l'Église romaine : Histoire de la mappemonde papistique sous le pseudonyme de Frangidelphe Escorche-Messe. Écrit par un protestant italien, Jean-Baptiste Trento, cet ouvrage attaque, d'une façon satirique, surtout les curés et les moines, sous l'angle de quatre thèmes reliés aux péchés capitaux : " la luxure traduite en paillardise, la gourmandise traduite par l'amour du ventre, la paresse dont l'oisiveté rend compte, l'avarice qui s'exprime par la simonie. Un terme générique désigne l'ensemble des curés et des moines dans le vocabulaire protestant, celui de « prestaille », ramassis de caphards, de cagots hypocrites, de cauteleux renards, voire de vénérables brigands » (278-279).

Sous la plume protestante, les moines sont traités de putiers, et les prêtres, de vilains putiers ! Dans l'escalade des injures, " putier » entraîne « putain », " putain » entraîne « bordel et maquereau » (« bordeau » au XVIe siècle), " bordel » entraîne " maquerelle ». Selon les protestants, les monastères ne sont que des bordeaux de moinerie comme le sont aussi les couvents de ces « nonnains toutes pollues », car, écrit Calvin, « il y a cent mille fois plus de vilenies aux cloistres des nonnains qu'en nul bordeau » (280).

La réplique catholique est d'appeler les protestants amateurs de " sensuelle liberté » et leur Église est également un «bordeau de prêtres et nonnains apostats, filles et femmes débauchées 
». Selon frère Beauxamis, Luther et Calvin ont avoué qu'ils se passeraient plutôt de manger que de femmes (281). En 1581, il dénonce la nouvelle Église calviniste qui s’allie avec les libertins voluptueux : « ... les porcs sardanapalistes ont converti la liberté offerte par Jésus-Christ en luxure : de leur ventre, ils ont fait leur dieu » (284).

Pour Calvin, les papes ne sont que de « bastards héritiers des Apôtres... »; quant à la papauté, il la caractérise de « comble et bourbe de toutes ordures et impiétez... » (300).

\section{Le vocabulaire bestial}

Protestants et catholiques, dans la polémique religieuse, se sont traités de noms d'animaux comme procédé littéraire d'attaque pour se dévaloriser les uns les autres. Voici un extrait de Postel (2004) que nous adaptons.

- Aigle : chez les catholiques, l'aigle symbolise la puissance orgueilleuse de Charles Quint, face au lys de France ; chez les protestants, l'aigle incarne la puissance orgueilleuse du pape qui fond sur sa proie, les réformés (343).

- Âne : aux yeux des protestants, l'âne symbolise les Sorbonistes, ignorants et entêtés dans leurs fausses interprétations de l'Écriture. Viret (Mét. I, 374) dit tout crûment des catholiques « les asnes seroyent meilleurs Chrestiens, Papes et Evesques qu'eux ». À leur tour, les catholiques comme Hangest, Doré ou Désiré, appellent Calvin « Calvin, asnier de Sathanas » (344-345).

- Babouin : Calvin dans les Actes du concile de Trente (1548) parle des pères conciliaires comme de « babouins qui n'ont nulle vergogne » (345).

- Bouc : symbole du péché. Pour Jean de Léry (Histoire mémorable, 1574), les huguenots sont " boucs ords et infâmes... Dignes avec Satan des infernales flammes ». Quant à Viret (Mét., II, 503), il s'écrie : « Dieu sait quels boucz sont aujourd'huy la plus grande part des Roys et princes »(346).

- Brebis : animal de prédilection des réformés (protestants) qui se sont reconnus dans cette sentence des Actes des Apôtres (Ac. 8, 32) «Comme une brebis, il a été conduit à la boucherie ». Quant aux catholiques, ils utilisent « brebis » en se référant maintes fois à la phrase de saint Matthieu (Mt. 7, 15) « Méfiez-vous des faux prophètes qui viennent à vous en habits de brebis mais sont loups ravissants (le loup étant le symbole de la voracité sauvage, la ruse) » (347).

- Chien : Beauxamis, dans sa Marmite renversée (6), traite les hérétiques (les réformés, les protestants) de « chiens retournant à leurs vomissements ». Calvin, dans les Actes du concile de Trente, dit de Monseigneur Du Prat qu'il va « flairant comme un chien de chasse les nidz de putains »(349).

- Corbeau : symbole de l'oiseau impur. Dans la polémique protestante, il désigne les prêtres. Ainsi, Luther, en 1540, parle de « ces corbeaux, le pape et les siens ». Mais Sorbin, dans Le Vray Resveille-Matin (1576), s'en prend aux réformés et s'élève contre « les outrageuses morsures de ces corbeaux » (350).

- Porc, pourceau : symbole de la luxure, de la goinfrerie, de la malpropreté, de la dépravation. « Pourceau » est l'invective la plus couramment employée par les deux camps. Que les citations émanent des « papistes » ou des réformés « hérétiques », elles utilisent des images semblables : moines ou ministres, sorbonistes ou luthériens vivent comme des pourceaux dans une étable couvent ou église -, c'est-à-dire qu'ils vivent dans la fange de leur vie dissolue (361). 
- Singe : cet animal est le symbole synthétique de tous les vices, gourmand, luxurieux, voleur, rusé. Plus que les catholiques, les protestants en ont fait grand usage, y compris dans ses formes dérivées "singeteau, singeon, singerie ", pour caractériser les curés, particulièrement dans l'exercice « de la messe, vraie singerie ». Dans le camp catholique, Saconay (Généalogie, 1573) préfère la femelle du singe, où il transforme « guenon » en « guenau » et « huguenot » en « huguenau », Calvin étant qualifié d' « archiguenau » (363).

- Vipère : symbole de la prudence, de la ruse, de la mort. Pour les catholiques et plus encore pour les protestants qui en font une lecture plus assidue, un verset des Psaumes dresse bien le portrait de « l'autre », de celui qui n'est pas de « votre » religion : " ils sont dévoyés dès le sein, les impies, égarés dès le ventre ceux qui disent l'erreur ; ils ont du venin de serpent, sourds comme l'aspic qui se bouche l'oreille... » (Ps. 58-4) (364).

Il est intéressant de noter que, dans ces échanges d'injures, où chaque camp traite l'autre de noms d'animaux, seul le terme « brebis » est utilisé dans deux sens complètement différents. En effet, les protestants, l'utilisent comme symbole pacifique auquel ils se comparent au point de se laisser simplement mener au bûcher ou à la boucherie. Quant aux catholiques, qui se réfèrent à un verset bien connu dans la Bible, ils qualifient les protestants de fausses brebis, c'est-à-dire de loups voraces et rusés sous une apparence de brebis.

\section{De femme... à putain}

Dans cette polémique du XVIe siècle et dans une France à $85 \%$ rurale, cette dernière « ...retient que Dieu a fait une « aide » à l'homme en la personne de la femme (Genèse 2, 15); son image reste celle d'un être mineur, sensuel, incapable de résister à la tentation... prête à toutes les paillardises, celles des chambrières et des nonnains dont savent si bien profiter ministres et curés. Et parce qu'elle n'a pas oublié son fautif désir de connaissance du temps paradisiaque, la femme se fait sorcière, copulant avec le Diable. Aussi ne faut-il pas s'étonner que le mot « putain » soit, de très loin, le plus utilisé par les pamphlétaires » (371).

Protestants et catholiques, à part les cas susmentionnés, ont généreusement utilisé « putain » pour s'insulter les uns les autres :

« - Moines effrontez comme putains de bordeaux (Viret, Désordre, 1545).

- Pape Clément...filz de putain (id.)

- Avant que les prestres de la Papauté ayant fourni tout ce qu'il faut à leurs putains et bastardz (Calvin, l'interim, 1549).

- Calvin, Farel, Viret... putains publiques (Cathelan, Passevent, 1556).

- Rome siège présidial des putains (Apologie pour Hérodote, 1566).

- Fils de putain de clergé (Mappemonde papistique, 1547).

- Catherine de Médicis... sale putain (L'estoile, 1576)» (490).

\section{De la signification de quelques injures}

Dans cette polémique religieuse du XVIe siècle, voici encore quelques extraits d'injures relevées par Postel (465-497), que catholiques et protestants proféraient les uns aux autres. Les injures étaient lancées soit contre les personnes, contre les objets sacrés, ou contre l'institution 
religieuse cible :

- Abominable Eglise romaine (Bade, Comédie, 1561).

- Abominables et exécrables ministres (Hervet, Ruses et finesses, 1562).

- L'abominable peste d'hérésie (Grenier, Bouclier de la Foy, 1568)

- Abominations papistes (Calvin, Libertins, 1545).

- Asnes (= ânes). Sorbonne qui engendre et nourrit les plus beaux asnes (Viret, Désordre, 1545).

- Luther, le gros bédier (= sot, ignare) (Désiré, Batailles et victoires, 1559).

- Ce petit belistre (= un rien du tout, voyou) (Gondi, Discours merveilleux, 1575).

- Beste (= bête). Cette grande beste en somme, c'est le pape de Rome (Chansons spirituelles II, 1555).

- Bordeau , bourdeau ( = bordel). Moynes en des bordeaux, ce sont leurs cloistres (1541, Institutions, Calvin). Ceste nouvelle Eglise est un bordeau (Beauxamis, Certains pourtraicts, 1564).

- Bougre (= sodomite, homosexuel). Jésuites, bougres et sodomites (Le cabinet, 1582).

- Bougrerie (= homosexualité, pratiques homosexuelles). Des livres qui enseignent non seulement paillardise mais aussi bougreries (Calvin, Censure, 1544).

- Brigand (= bandit). Brigands romains (Luther, De la papauté de Rome, 1520).

- Canaillerie (= malhonnêteté). Canaillerie spirituelle et mondaine (Luther, De la papauté de Rome, 1520).

- Carbonnadable (= susceptible d'être réduit en charbon, en cendres). Hérétiques, bruslables, fricassables, rotissables et carbonnadables (Marnix, Différens, 1599).

- Catin. Pontifes... catins et prostituées de la plus belle eau (Bèze, Passavant, 1553).

- Chancre. La parole des hérétiques procède comme un chancre (Hangest, En controverse, 1536).

- Charongne (= charogne). Cette charongne s'ose comparer à Siméon (1548, Les Actes du Concile, Calvin). [Les curés] cherchent et suyvent les charongnes (Viret, Satyres, 1560).

- Coquin (= vaurien). Les coquins romains (Luther, De la papauté de Rome, 1520).

- Coquinaille (= assemblée de coquins). Le Pape et les siens tous / Maudite coquinaille (Bordier, Chanson).

- Corbeau (= homme sans scrupules; aussi : prêtres). Ces corbeaux, le pape et les siens (Luther, Les faits de J.-C., 1540). Vous eussiez veu tous les autres moines comme corbeaux sur une charogne s'assembler, corbiner (= croasser) (Marnix, Différens, 1599).

- Cul. Bouche du Pape et cul du Diable, c'est tout un (Bèze, Passavant, 1553).

- Enragé. Ces bestes enragées [de la Sorbonne] (Calvin, Censure, 1544).

- Excrément. [Évangiles de l'Antéchrist] excréments que le diable a vomi par la bouche fétide des Papes (Bèze, Passavent, 1533) - Les jésuites... les plus vils excréments de la Papauté (Daneau, Antéchrist, 1572).

- Exécrable. Exécrable marchandise et foyre des messes (Calvin, Épître à Sadolet, 1540). [Cordeliers] exécrable couvée de Satan (Bade, Alcoran, 1560).

- Fallourdier (= prêtre vivant de menus profits). Les moynes fallourdiers (Calvin, Actes du Concile, 1548). 
- Fangeux : abject. Pourceaux fangeux (Balade des luthériens, 1529).

- Fiente (= excrément d'oiseau). La fiente et le bourbier ne flaire point mal aux pourceaux (Calvin, Censure, 1544) - [Hérétiques] juments qui pourissent en leur fiente (Beauxamis, Marmite renversée, 1581).

- Fripon. Fripons... romains (Luther, De la papauté de Rome, 1520). - Luther fripon masqué (Benoist, Discours en forme de dialogue, 1570).

- Galafre, galaffre, galiffre (= homme débauché). "Grand galiffre » désigne le Pape : c'est le grand prevost des marchans... convertissant le plomb en or (Marcourt, Le livre des marchans, 1533) - Pour servir à ce grand galiffre (Viret, Satyres, 1560).

- Immondice (= ordure). [Calvin] esgout et retraict de toutes les immondices des hérétiques (Du Val, Mirouer des calvinistes, 1562).

- Infâme (= abject). Damnez infâmes (Apologie pour Hérodote, 1566) - Prestres n'êtes-vous pas infâmes (Bordier, Chanson, 1532).

- Infect (= répugnant). La messe infecte (Bordier, Chanson, av. 1555).

- Infernal (= qui est de l'enfer). Maudicte furie infernale(La Vacquerie, Catholique remonstrance, 1560). Ce Cacus, monstre infernal (Bade, Comédie, 1561).

- Ivrogne (= ivrongne, yvrogne) : surtout appliqué aux prêtres et à toute l'Église romaine. [Curés] les plus grands yvrongnes de la terre (Calvin, Censure, 1544).

- Lépreux. Doctrine lépreuse de Luther (Désiré, Les disputes..., 1559).

- Loup (= voracité, ruse). Grand loup romain (Bade, Alcoran, 1560). Loups desguisez (Pasquier, Complainte de l'Université, 1564). Loup-papal (Bordier, Chanson, 1565).

- Malignan (= malfaisant). Eglise des malignans : assemblée des iniques (Lambert, Somme chrestienne, 1529).

- Maquereau (= proxénète). Ces beaux Evesques de l'Antéchrist sont semblables aux maquereaux, larrons et brigans (Bade, Alcoran, 1560) - Certains cardinaux bons maquereaux (Ibid.).

- Maquerelage (= prostitution organisée). S'en servir [de l'Évangile] à faire un maquerelage pour gagner quelques bénéfices (Calvin, Nicodémites, 1544).

- Mauvaisie (= méchanceté, vice). La mauvaisie des Papes (Lemaire, Schismes et conciles, 1562).

- Ordure (= saleté). Infecter la compagnie de leur ordure (Calvin, Anabaptistes, 1544). L'ordure d'hérésie (Hervet, Purgatoire, 1562). Cloaques et ordures de la chrestienté (Cabinet du roy, 1582).

- Paillard, paillar, paillart (= débauché). La grande paillarde Eglise papale (Mandement de Lucifer, 1562).

- Paillardise (= débauche). Vos apostaz... en leur paillardise (Désiré, Contre-poison, 1562). Pontificat, siège de paillardise (Le rasoir, 1562).

- Pape. Ce beau diable de Pape (Mandement de Lucifer, 1562). Tant de saintcz papes, gaillards papillons (= haut clergé), papegeaux et paillards (Marnix, Différens, 1599).

- Papillon (= haut clergé). Nos Papes et papillons s'entretiennent l'un l'autre par le cul comme hannetons (Marnix, Différens, 1599).

- Papistique (= qui relève des papistes (= qui relève du pape)). Crapules papistiques (Le rasoir, 
1562).

- Peste. [Le Pape] peste de l'Eglise (Von Hutten, Julius, 1513). L'abominable peste d'hérésie (Grenier, Bouclier de la foy, 1568) - La très pernicieuse peste d'hérésie (Benoist, Discours en forme de dialogue, 1570).

- Pestifère, pestilentieux (= qui donne la peste). Luther qui a vomi son pestifère...venin (Gacy, Trialogue, 1524). [De Luther] pestilentieuse doctrine (Du Val, Mirouer des calvinistes, 1559). Entreprises pestilentieuses de ceste nouvelle secte (Beauxamis, Marmite renversée, 1581).

- Pollu (= souillé). Le Pape et sa secte pollue (Bordier, Chanson, 1546).

- Pourceau (= saleté, gourmandise, luxure). [Luthériens] vivent ainsi que ung toict les pourceaulx (Gringore, Blazon, 1524).

- Propligateur (= destructeur). St Hilaire propligateur des hérétiques Ariens (Beauxamis, Marmite renversée, 1581).

- Puant. Ce chien estant sorty de la fange de son puant cloistre (Calvin, Actes du Concile, 1548).

- Puantise (= mauvaise odeur). La puantise du pape Paul (Luther, Les faits de J.-C., 1540).

- Putain (= prostituée). Putains auront toujours l'honneur dedans Rome (Meylan, Coq à l'âne, 1536). Moines effrontez comme putains de bordeaux (Viret, Désordre, 1545). Pape Clément... filz de putain (id.). Calvin, Farel, Viret... putains publiques (Cathelan, Passevent, 1556). Rome siège présidial des putains (Apologie pour Hérodote, 1566).

- Retraict, retrait, retret (= toilettes). [L'Église] puant retraict de Satan (Luther, Antithèse, 1545). Quand il y aurait un retraict de quelque sainct, il le faudrait honorer (Calvin, Sacrée faculté, 1545).

- Ribaudaille (= troupe de femmes débauchées). Pas n'ont soucy ces gros paillars (les prêtres) de nourrir leur ribaudaille (Marcourt, Déclaration de messe, 1534). Evesques et Abbez, ce n'est que ribaudaille (Bordier, Chanson).

- Ruffian, ruffien (= voyou, entremetteur). [Les moines] font leur triomphe d'être cogneuz bons ruffiens (Calvin, Scandales, 1550).

- Sathan, satan (= satan). [L'Église] paillarde de Satan (Luther, Antithèse, 1545). Secte sathanique (Beauxamis, Marmite renversée, 1581).

- Sodomite (= qui pratique la sodomie). Ces papes sodomites (Bèze, Passavant, 1553).

- Symoniacle (= simoniaque, celui qui vend une chose spirituelle). Gens d'églises symoniaques. Damnez symoniacles (Bouchet, Déploration, 1572).

- Synagogue (= assemblée, réunion. S'applique à l'Église romaine et aux Églises réformées). Synagogue de Sathan (Ochino, L'image de l'Antéchrist, 1544). Ceste ribaude fille de sinagogue (Viret, Désordre, 1545).

- Théologastre (= péjoration de théologien; théologien du ventre : allusion à la réputation de goinfrerie du clergé). Les théologastres papistes (Calvin, Scandales, 1550).

- Vermine. Le Pape et toute sa vermine de Cardinaux (Marcourt, Conclusion de la messe, 1563).

Dans ce chapelet d'injures précédent, « putain » est l'injure en écho par excellence que catholiques et protestants se lancent avec un synonyme « catin »; «putain »s'applique aussi bien aux êtres animés qu'inanimés. 
Également, sur ces vocables injurieux se brodent des actions non élogieuses de l'adversaire : ces jolis puceaux (prêtres ou moines catholiques) gardent leurs licts vuydes d'une seule femme afin qu'ils ayent congé d'envahir tous les litz des gens mariez; Bèze (protestant) engrosse une chambrière qu'il fait avorter; nos papes et papillons (haut clergé) s'entretiennent l'un l'autre par le cul...

\section{Structures phrastiques des injures}

Sur le plan syntaxique, les injures prennent généralement les formes suivantes :

1- L'injure exclamative ou l'exclamation de dépréciation. Exemple : Quel imbécile ! Quel salaud! Quel crétin! Quelle crapule !

2- L'injure en tant qu'attribut. Exemple : Cet évêque est un salaud.

3- L'injure en « enchâssement ». Exemple : Cet imbécile de salaud de crétin de curé.

À noter que le terme de référence «curé » se trouve à la fin du cortège nominal d'injures.

4- L’injure nominale en dislocation. Exemple : Il a abandonné le froc, l'imbécile.

5- L'injure dans une relative à la première ou à la deuxième personne. Exemples : Imbécile que je suis. Pauvre imbécile que tu es.

6- L'injure comparative en gradation. Exemples : Il est plus stupide que le roi.

7- L'injure avec marqueur de quantité (si, assez) et corrélateur de conséquence. Exemples : Elle est si avare qu'elle vendrait sa mère. Il est si naïf que tu pourrais lui faire croire n'importe quoi.

8- L'injure en gradation : un des moyens pour rendre l'injure plus blessante consiste à adjoindre à l'injure nominale un adjectif. Cet adjectif, curieusement neutre ou peu sympathique, ne fait que renchérir et renforcer l'effet de l'injure. Exemple :

« C'est un grand imbécile » ne veut pas dire un imbécile qui est grand mais un un vrai imbécile ; autrement dit, l'injure « imbécile » se trouve accentuée par l'adjectif « grand ».

Qu'en est-il de l'escrime verbale ou scripturaire entre catholiques et protestants ?

Nous remarquons que les structures les plus fréquentes sont celles qui se rapportent à :

- l'injure en tant qu'attribut ou épithète : les uns sont apostats... les autres putiers ; luthériens réprouvés et idiotz; moines traistres, moines paillards, moines vermines, papes sodomites, prêtres vilains putiers, prêtres cafards, prêtres cagots, prêtres hypocrites, vénérables brigands,

- l'injure en gradation se présente :

a) soit comme un nom déjà péjoratif accompagné d'un adjectif qui le renforce : apostats infâmes, abominables ténébrions, pervers calomniateurs, misérable bestiole, grand paillard, cauteleux renards, libertins voluptueux.

b) soit comme un groupe nominal suivi d'un complément de nom : moines de nature servile, amateurs de sensuelle liberté, bordeau (bordel) de prêtres, papauté bourbe d'ordures, bastards héritiers des Apôtres.

- l'injure en enchâssement : moines putains de bordeaux (bordels) ; fils de putain de clergé.

- l'injure comparative : à part l'abondance de comparaisons de l'adversaire au répertoire bestial (âne, babouin, chien, corbeau, porc, singe, vipère...), certaines comparaisons se présentent dans une structure moins familière comme « bouche du Pape et cul du Diable c'est tout un »; les asnes seroyent meilleurs Chrestiens, Papes et Evesques qu'eux (les catholiques); il y a cent mille fois plus de vilenies aux cloistres des nonnains qu'en nul bordeau; leurs églises sont pires que des 
étables de pourceaux.

- l'injure en écho : si un camp traite l'adversaire de «putain », le camp adverse renvoie l'injure comme telle, voire plus brodée ou assaisonnée. Il en est de même avec l'expression « bordeau (bordel) de prêtres et nonnains apostats ».

- l'injure antithétique : notre Sainte Mère putain l'Eglise romaine, les cloîtres bordeaux, vénérables brigands, monastères bordeaux de moinerie, la grande paillarde Eglise papale.

Bien que nous nous concentrions sur la polémique religieuse au XVIe siècle en France, nous nous permettons de citer, dans l'Angleterre du XVIIe siècle lors de la répression sévère de la propagande catholique, les «vers jésuitiques » suivants que nous empruntons à Lecercle (1996, 84), lesquels reflètent créativité et sarcasme avec une douce violence :
I hold for sound faith
What England's church allows
What Rome's faith saith
My conscience disavows

Ces vers provenant de Wells (1963) et repris par Lecercle démontrent comment la langue peut être manipulée pour servir l'intention de leurs auteurs. En effet, ces vers lus horizontalement sont anglicans et donnent :
I hold for sound faith
What England's church allows
What Rome's faith saith
My conscience disavows

Lus verticalement en deux rangées séparées, ces mêmes vers deviennent catholiques :

I hold for sound faith

What Rome's faith saith
What England's church allows

My conscience disavows

\section{Conséquences des injures}

Pour l'insulteur, selon Dulude et Trait (1991), des études avancées (aucune référence bibliographique) permettent d'établir que le fait d'injurier quelqu'un décongestionne le foie et active l'élimination de la bile; facilite l'assimilation de l'azote et de l'oxygène ; fortifie le système cardiaque, stimulant la circulation du sang; fait abondamment transpirer, favorisant ainsi l'élimination de la cellulite ; et surtout détend énormément les nerfs (11).

Pour l'insulté, rien n'est dit. Cependant, si ce qui précède est médicalement prouvé, il faudra que l'insulté devienne insulteur ! Une question alors se pose : quand la véritable communication va-t-elle s'établir?

Dans l'étude que nous avons présentée, l'histoire nous indique que les antagonistes ne se sont pas tout simplement échangé des injures mais se sont également entretués. Est-il nécessaire de savoir si les propos injurieux précédaient ou suivaient leurs luttes sanglantes. Dans les deux cas, les injures, qu'elles aient été éléments déclencheurs ou éléments conséquents, ont indubitablement contribué à attiser le feu. Nous pourrions dire, dans le contexte précédent, avec Lecercle (1996) : le rôle du langage n'est pas de transmettre de l'information mais du désir (...) et de la violence (...) (270). 


\section{CONCLUSION}

Catholiques et protestants ont bien exploité toute la gamme des injures contre des individus nommément, contre des groupes et leurs pratiques, contre les objets sacrés et l'Église elle-même : ironie, injures agressives ou blessantes, grossières ou obscènes, ordurières ou scatologiques, odieuses ou méprisantes, bestiales ou sexistes, vulgaires ou sarcastiques.

Loin de simplifier les raisons politico-religieuses sous-jacentes aux propos acerbes et belliqueux, nous avons voulu dévoilé un triste tableau où l'on passe de la violence verbale à l'acte meurtrier entre catholiques et protestants au XVIe siècle. Pour bien saisir la portée des injures entre les deux camps, il nous importait de citer de nombreux exemples afin de relever, en plus de la structure phrastique des injures, leurs justes valeurs sémantiques en sachant qui dit quoi à qui et dans quelle intention : les protestants à la fois attaquent l'institution ecclésiastique et se défendent contre la répression; les répliques catholiques dévoilent la colère d'une institution ecclésiale qui se voit bafouée et accablée d'invectives.

Quelles que soient les expressions injurieuses débitées, honnir semble avoir été une stratégie privilégiée des deux camps puisque vouer l'adversaire au mépris public en le couvrant de honte était susceptible de s'attirer des partisans. Les catholiques avaient comme thème de prédilection dans leurs pamphlets « prier Dieu que les assemblées secrètes et congrégations de tels libertins soient abattues... puis tous ensemble mettre à feu et à sang telle secte de Bannis et pleins de vice » et vouer les protestants à l'anathème. Quant aux protestants, en plus des injures forgées, ils avaient surtout recours aux injures bestiales, pourvues de dérision et de sarcasme pour avilir et discréditer l'adversaire. Contrairement à l'opinion contemporaine qui veut que la pratique de l'injure soit parfois un défoulement et une saine hygiène mentale, au XVIe siècle, elle était, entre autres, au service de la haine, de l'avilissement et de la destruction de l'autre.

\section{BIBLIOGRAPHIE}

Bénac, Henri. Dictionnaire des synonymes. Paris : Hachette, 1956.

Crouzet, Denis. Les guerriers de Dieu. Paris : Champ Vallon, 1990.

Doillon, Albert. Le dico de la violence. France : Arthème Fayard, 2002.

Dulude, Yvon, et Jean-Claude Trait. Dictionnaire des injures québécoises.

Québec : Stanké, 1991.

Édouard, Robert. Nouveau dictionnaire des injures. France : Sand, 1983.

Lecercle, Jean-Jacques. La violence du langage. Paris : Presses universitaires de France, 1996.

Lécrivain, Philippe. «Violences et religions en France à la fin du XVIe siècle. » Centre de recherche et d'actions sociales 28 (juillet, 2004) : 1-2.

Milner, Jean-Claude. De la syntaxe à l'interprétation. Quantités, insultes, exclamations. Paris : Seuil, 1978.

Robert, Paul. Dictionnaire Le Petit Robert. Paris : Dictionnaires Le Robert, 1993

Postel, Claude. Traité des invectives. Paris : Société d'édition Les Belles Lettres, 2004. 